\title{
LSD1-S112A exacerbates the pathogenesis of CSE/LPS-induced chronic obstructive pulmonary disease in mice
}

\author{
Jiyeong Jeong ${ }^{1,2}$, Chaeyoon $\mathrm{Oh}^{3}$, Jiwon $\mathrm{Kim}^{3}$, Chul-Gyu $\mathrm{Yoo}^{2, *}$ \& Keun $\mathrm{Il} \mathrm{Kim}^{1,3, *}$ \\ ${ }^{1}$ Research Institute of Women's Health, Sookmyung Women's University, Seoul 04310, ${ }^{2}$ Department of Internal Medicine, Seoul National \\ University College of Medicine, Seoul 03080, ${ }^{3}$ Department of Biological Sciences, Cellular Heterogeneity Research Center, Sookmyung \\ Women's University, Seoul 04310, Korea
}

\begin{abstract}
Lysine-specific demethylase 1 (LSD1) is an epigenetic regulator that modulates the chromatin status, contributing to gene activation or repression. The post-translational modification of LSD1 is critical for the regulation of many of its biological processes. Phosphorylation of serine 112 of LSD1 by protein kinase $C$ alpha (PKC $\alpha$ ) is crucial for regulating inflammation, but its physiological significance is not fully understood. This study aimed to investigate the role of Lsd1-S112A, a phosphorylation defective mutant, in the cigarette smoke extract/LPS-induced chronic obstructive pulmonary disease (COPD) model using $L s d 1^{S A S A}$ mice and to explore the potential mechanism underpinning the development of COPD. We found that $L s d 1^{S A / S A}$ mice exhibited increased susceptibility to CSE/LPS-induced COPD, including high inflammatory cell influx into the bronchoalveolar lavage fluid and airspace enlargement. Additionally, the high gene expression associated with the inflammatory response and oxidative stress was observed in cells and mice containing Lsd1-S112A. Similar results were obtained from the mouse embryonic fibroblasts exposed to a PKC $\alpha$ inhibitor, Go6976. Thus, the lack of LSD1 phosphorylation exacerbates CSE/LPS-induced COPD by elevating inflammation and oxidative stress. [BMB Reports 2021; 54(10): 522-527]
\end{abstract}

\section{INTRODUCTION}

Cigarette smoking is a critical risk factor for the development

*Corresponding authors. Keun Il Kim, Tel: +82-2-710-9768; Fax: +82-2-2077-7322; E-mail: kikim@sookmyung.ac.kr; Chul-Gyu Yoo, Tel: +82-2-2072-3760; Fax: +82-2-2072-0630; E-mail: cgyoo@snu.ac.kr

https://doi.org/10.5483/BMBRep.2021.54.10.034

Received 4 March 2021, Revised 25 March 2021, Accepted 25 May 2021

Keywords: Cigarette smoke extract, COPD model, Inflammation, LSD1 phosphorylation, Oxidative stress and progression of chronic obstructive pulmonary disease (COPD), characterized by chronic bronchitis and emphysema (1). Cigarette smoke (CS) induces inflammatory responses and oxidative stress by altering the cellular redox status, resulting in epigenetic modifications. Several studies have demonstrated that epigenetic enzymes including DNA methyl-transferase, histone (de)acetylase, and histone (de)methylase, act as crucial regulators of COPD pathogenesis (2). For example, suppression of Histone deacetylase 2 (HDAC2) increases pro-inflammatory cytokine production through NF-KB signaling in CS-exposed cells and patients with COPD (3). Histone deacetylase SIRT1regulated expression of matrix metalloproteinase 9 is associated with inflammation and lung remodeling in patients with COPD (4). Although epigenetic modifiers are widely reported to be involved in the regulation of COPD pathogenesis (5), the role of histone demethylases in this phenomenon is not well studied. Recent studies revealed that the expression of USP38, a potential deubiquitylating enzyme of lysine-specific demethylase 1 (LSD1), also known as KDM1A, is upregulated in the COPD lung tissue. USP38 enhances the stability of LSD1, thereby activating signaling pathways and promoting cell proliferation and colony formation $(6,7)$, which suggests a role of LSD1 in COPD pathogenesis.

LSD1 is a member of the flavin adenine dinucleotidedependent amine oxidase family (8). LSD1 was first isolated as an interaction partner of the histone deacetylase HDAC2 and identified as a histone demethylase (9). LSD1 specifically removes methyl groups from histone $\mathrm{H} 3$ lysine 4 and $\mathrm{H} 3$ lysine 9, and thus, can operate as a transcriptional repressor or activator, respectively (10). Furthermore, LSD1 can demethylate and regulate the function and stability of non-histone proteins such as p53, DNMT1, HIF-1 $\alpha$ and p65, which are involved in gene expression (11). Recent evidence showed that several kinases, such as PKC $\alpha$, PLK1, CK2 and GSK3 $\beta$, phosphorylate serine residues in LSD1 and thereby, regulate its biological functions (12-16). For example, PKC $\alpha$-mediated phosphorylation of LSD1 on S112 regulates diverse biological processes including circadian rhythm, presynaptic plasticity, epithelialmesenchymal transition, and inflammatory responses $(12,15$,

ISSN: 1976-670X (electronic edition)

Copyright (c) 2021 by the The Korean Society for Biochemistry and Molecular Biology

(c) This is an open-access article distributed under the terms of the Creative Commons Attribution Non-Commercial License (http://creativecommons.org/licenses/by-nc/4.0) which permits unrestricted non-commercial use, distribution, and reproduction in any medium, provided the original work is properly cited. 
17 , 18). Furthermore, phosphorylation of LSD1 by $\mathrm{PKC} \alpha$ represents a crucial mode of epigenetic regulation in inflammatory diseases, such as sepsis and inflammatory bowel disease in murine models $(15,19)$. However, little is known about the precise role of LSD1 in CS-induced pulmonary inflammation in the COPD model.

In this study, we explored the effects of Lsd1-S112A on the inflammatory response and oxidative stress in the COPD model using phosphorylation defective mutant Lsd1-S112A $\left(L s d 1^{S A / S A}\right)$ mice.

\section{RESULTS}

\section{$L s d 1^{S A / S A}$ mice are susceptible to CSE/LPS-induced COPD}

To investigate the role of Lsd1-S112A in the onset of COPD in vivo, as an initial approach, WT and $L s d 1^{S A / S A}$ mice were injected intratracheally with CSE-only, LPS-only, or CSE and LPS (CSE/LPS), which are well-established approaches for developing COPD in mice (Fig. $1 \mathrm{~A}$ ). $W T$ and $L s d 1^{\text {SA/SA }}$ mice treated with CSE or LPS showed COPD-like lung pathology, characterized by immune cell infiltration and airspace enlargements. Histopathological examinations revealed that CSE-only and LPS-only injections triggered lung injury and airspace enlargements in both $W T$ and $L s d 1^{S A / S A}$ mice (Fig. 1B). This indicated that CSE and LPS can individually induce emphysema in mice. Airspace enlargement was evaluated by comparing the mean linear intercept (MLI). MLI scoring revealed significant airspace enlargements in $L s d 1^{S A / S A}$ mice than in WT littermates, when comparing the vehicle control, CSE, or CSE/LPS groups independently (Fig. 1C). The difference in the MLI scores between the $W T$ and $L s d 1^{\text {SA/SA }}$ mice was greater in the CSE-only group than in the LPS-only group (Fig. 1C). In addition, histological images showed that the infiltration of inflammatory cells around the airways and vessels was induced by CSE and LPS treatment (Fig. 1D), and airway inflammation score, which is evaluated by scoring the presence of peribronchial and perivascular inflammation, was higher in $L s d 1^{S A / S A}$ mice compared with WT mice when CSE/LPS was administered (Fig. 1E).

To address whether $L s d 1^{S A S A}$ mice exhibit increased inflammation, we evaluated the immune cells in the bronchoalveolar lavage fluid (BALF) one day after the last dose of CSE or LPS in $W T$ and $L s d 1^{S A / S A}$ mice (Fig. 2A). Cells in the BALF were stained with Diff-Quik and the total number of cells was evaluated. Representative images of cells in the BALF from each group are shown in Fig. 2B. LPS-injected $W T$ and $L s d 1^{S A / S A}$ mice exhibited a marked increase in immune cell infiltration in BALF (Fig. 2C), consisting mainly of macrophages and neutrophils (20). In the CSE/LPS-injected $L s d 1^{S A / S A}$ mice, the number of infiltrated immune cells increased by 2.7 -fold compared with those in WT mice, reaching levels observed in both $W T$ and $L s d 1^{S A / S A}$ mice injected with LPS-only, but the increased level was much smaller in WT mice (Fig. 2C). Consistently, the number of neutrophils infiltration, the major infiltrating cell type, increased by 6.0 -fold in $L s d 1^{S A / S A}$ mice than in WT mice when treated with CSE/LPS (Fig. 2D). CSE has immunosuppressive effects leading to impairment of antimicrobial defense mechanisms in the airway epithelium. Neutrophil recruitment factors released by LPS treatment, such as GM-CSF and IL-8, are known to be reduced following CSE exposure (21). Taken together, we found that $L s d 1^{S A / S A}$ mice were more susceptible to CSE/LPS-induced lung damage and inflammatory responses, compared with those seen in WT mice.

\section{Effect of $L s d 1^{S A / S A}$ on Cox-2 and Ho-1 expressions in CSE/LPS-induced COPD}

Exposure to CSE promotes the pathogenesis of COPD by increasing oxidative stress and inflammation in the lung (22). To examine whether the phosphorylation status of LSD1 affects airway inflammation and oxidative stress following CSE and LPS exposure, we compared the expression of Cox-2 and
A

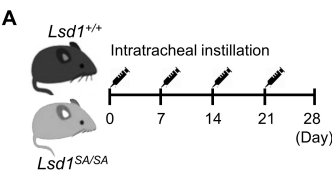

C

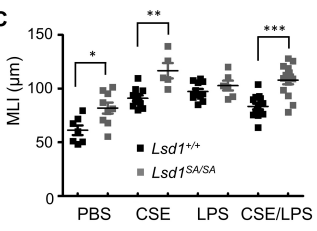

D

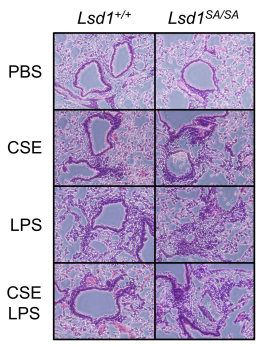

B

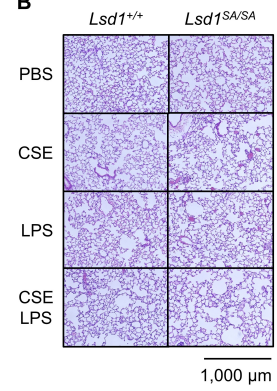

E

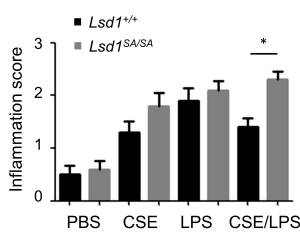

Fig. 1. $L s d 1^{S A / S A}$ mice are highly susceptible to CSE/LPS-induced COPD. (A) Schematic diagram of the animal model for chronic obstructive pulmonary disease (COPD) induced by cigarette smoke extract (CSE) combined with lipopolysaccharide (LPS) in mice. WT and $L s d 1^{S A / S A}$ mice were subjected to intratracheal instillation of vehicle (100 $\mu \mathrm{l} /$ mouse, $\mathrm{n}=8)$, CSE (100 $\mu \mathrm{l} /$ mouse, $\mathrm{n}=5)$, LPS $(10 \mu \mathrm{g} /$ mouse, $\mathrm{n}=6)$, or CSE/LPS $(10 \mu \mathrm{g}$ LPS in $100 \mu \mathrm{l} \mathrm{CSE} /$ mouse, $\mathrm{n}=14$ ) on the indicated days. (B) Representative histopathological images of lung tissues stained by Hematoxylin and Eosin (H\&E) or (C) Mean linear intercept (MLI) scoring from WT and $L s d 1^{\text {SAISA }}$ mice. MLI values are expressed in $\mu \mathrm{m}$. Scale bar, $1000 \mu \mathrm{m}$. All results represent three independent experiments. Data are presented as mean $\pm \mathrm{SEM} ; * \mathrm{P}<0.05, * * \mathrm{P}<0.01$, ***P $<0.001$. (D, E) Representative histological images are shown at 200x magnification. Airway inflammation score was defined as the average of the peribronchial and perivascular inflammation scores. $n=4$ per group; data reported as mean $\pm \mathrm{SEM} ; * \mathrm{P}<0.05$. 


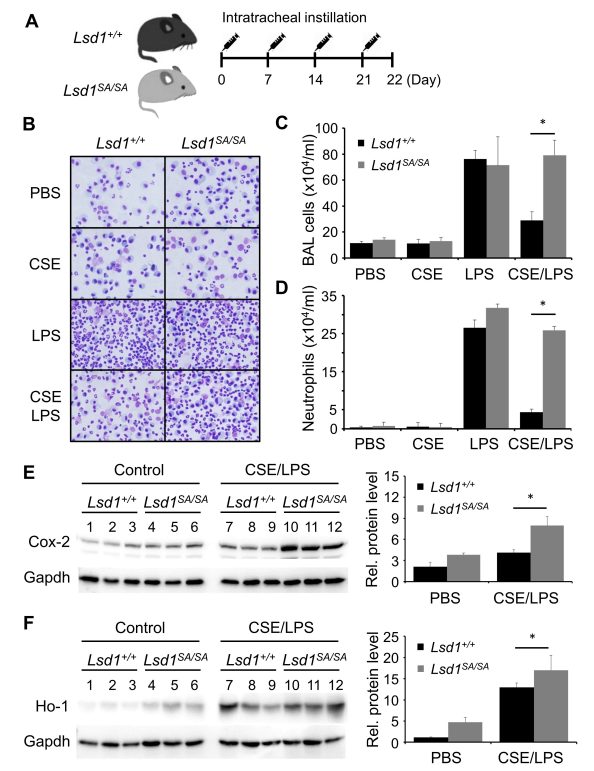

Fig. 2. Analysis of CSE/LPS-induced inflammatory and oxidative stress responses in $L s d 1^{S A / S A}$ mice. (A) Schematic diagrams of murine COPD model for analyzing the number of cells in BALF (B) Photomicrographs of Diff-Quik-stained cells are shown at 200x magnification and $(C)$ total cells and (D) neutrophils count in BALF from the indicated treatment groups. $n=6$ per group; data are presented as mean \pm SEM; $* P<0.05$. (E, F) Immunoblot analysis was performed using the indicated antibodies in lung homogenates. Lane 1-3: control group in $\mathrm{Lsd1^{+/ }}$ mice, Lane 4-6: control group in $L s d 1^{S A / S A}$ mice, Lane 7-9: CSE/LPS group in $L s d 1^{+/+}$mice, Lane 10-12: CSE/LPS group in $L s d 1^{S A / S A}$ mice. The immunoblots were quantified using Imagej. $\mathrm{n}=6$ per group; data are presented as mean $\pm \mathrm{SEM} ; * \mathrm{P}<0.05$.

Ho-1 in the $W T$ and $L s d 1^{S A / S A}$ lung tissues from each group. We quantified the Cox-2 and Ho-1 protein levels by immunoblotting and densitometry. When treated with CSE/LPS, Cox-2 levels were significantly upregulated in $L s d 1^{S A / S A}$ mice compared with the level in WT mice (Fig. 2E). Ho-1 expression was also upregulated in $L s d 1^{S A / S A}$ mice upon CSE/LPS treatment (Fig. 2F). These results indicate that Lsd1 phosphorylation on S112 is related to the regulation of Cox-2 and $\mathrm{Ho}-1$ expression upon CSE/LPS exposure in vivo. Taken together, our in vivo data suggest that Lsd1-S112A exacerbates inflammation and oxidative stress in CSE/LPS-induced COPD.

\section{Increased levels of oxidative stress biomarkers in $L s d 1^{S A / S A}$ MEFs exposed to CSE and LPS}

Although we detected elevated expression of Cox-2 and Ho-1 in the lungs of $L s d 1^{S A / S A}$ mice compared with that in WT mice upon CSE/LPS treatment, the in vivo data is the final outcome of combinatorial signaling. To investigate whether Lsd1-S112A directly affects inflammation and oxidative stress, we compared the pattern of Cox-2 and Ho-1 expression in WT and $L s d 1^{\text {SA/SA }}$ MEFs following CSE and LPS exposure. MEFs were treated

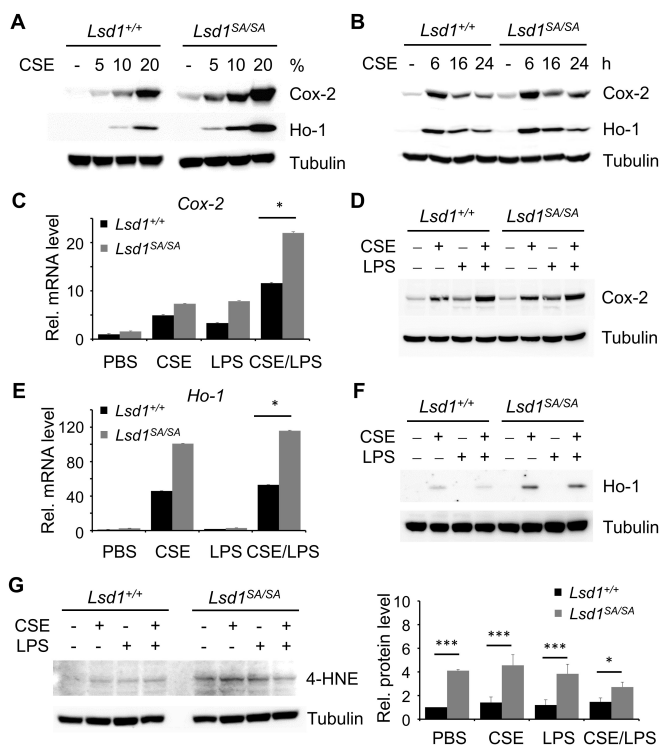

Fig. 3. Increased biomarkers of oxidative stress in $L s d 1^{\text {SA/SA }}$ MEFs exposed to CSE and LPS. (A, B) Cox-2 and Ho-1 protein levels in $W T$ and $L s d 1^{S A / S A}$ MEFs challenged with CSE at the indicated dose for $24 \mathrm{~h}$ or $10 \%$ CSE for the indicated time periods, respectively, were assessed by western blotting. MRNA and protein levels of Cox-2 (C, D) and Ho-1 (E, F) in WT and Lsd1 ${ }^{\text {SA/SA }}$ MEFs were shown after treatment with CSE and LPS for $4 \mathrm{~h}$ or $24 \mathrm{~h}$, respectively. All results represent three independent experiments. Data are presented as mean \pm SEM; ${ }^{* P}<0.05$. (G) 4-HNE-modified protein was used as an oxidative damage marker. The immunoblots were quantified using ImageJ. Graph was obtained from five independent experiments. Data are presented as mean \pm SEM; $* P<0.05, * * * P<0.001$.

with CSE at the indicated doses and time points, and the cell lysates were used for immunoblotting. The Cox-2 and Ho- 1 protein levels showed a dose-dependent increase upon CSE treatment, which was further enhanced in $L s d 1^{\text {SA/SA }}$ MEFs at a high concentration of CSE (i.e., 20\%) compared with those in WT MEFs (Fig. 3A). The time-course analysis also exhibited higher and longer expression of Cox-2 and Ho-1 in $L s d 1^{\text {SA/SA }}$ MEFs compared with that in WT MEFs (Fig. 3B). Thus, these results confirm in vivo data that $L s d 1^{S A / S A}$ mice are more sensitive to CSE/LPS treatment with increased expression of Cox-2 and Ho-1.

WT and $L s d 1^{S A / S A}$ MEFs were then treated with CSE, LPS or CSE/LPS for $4 \mathrm{~h}$ or $24 \mathrm{~h}$ to assess the mRNA and protein levels of Cox-2 and Ho-1. The Cox-2 mRNA and protein levels were higher in $L s d 1^{\text {SAISA }}$ MEFs compared with WT MEFs in each group (Fig. 3C, D). Interestingly, elevated expression of Ho-1 in $L s d 1^{\text {SAISA }}$ MEFs, compared with WT MEFs, was specific to the groups containing CSE (i.e., CSE and CSE/LPS group) (Fig. 3E, F). 4-Hydroxy-2,3-trans-nonenal (4-hydroxynonenal, 4-HNE), the aldehydic product of lipid peroxidation, is widely accepted as a reliable marker for oxidative stress (23). WT and $L s d 1^{\text {SA/SA }}$ 
MEFs were treated with CSE, LPS or CSE/LPS for $24 \mathrm{~h}$ to assess the cellular levels of 4-HNE-modified proteins as an indicator of oxidative stress. Immunoblot analysis revealed higher levels of 4-HNE-modified proteins in $L s d 1^{\text {SA/SA }}$ MEFs compared with WT cells in all groups (Fig. 3G). 4-HNE is a cytotoxic product of lipid peroxidation and acts as signaling molecules. It has been uncovered as a factor causing pathological changes under oxidative stress and a key mediator of oxidative stressinduced cell death (24). Therefore, it is likely that Lsd1-S112A MEFs are intrinsically more sensitive to oxidative stress.

\section{Effect of Go6976 on CSE/LPS-induced Cox-2 and Ho-1 expression in MEFs}

To validate the hypothesis that the phosphorylation defect of Lsd1-S112 is critical for the expression of Cox-2 and Ho-1 proteins in MEFs, we used Go6976, a PKC $\alpha$ inhibitor, to block the phosphorylation of Lsd1-S112. Immunoblot analysis was performed on MEF extracts following $24 \mathrm{~h}$ CSE and LPS treatment in the absence or presence of Go6976. Interestingly, pre-treatment with Go6976 enhanced Cox-2 and Ho-1 expression (Fig. 4A, B). In a separate experiment, MEFs were treated with Go6976 or vehicle for $1 \mathrm{~h}$ and then treated with
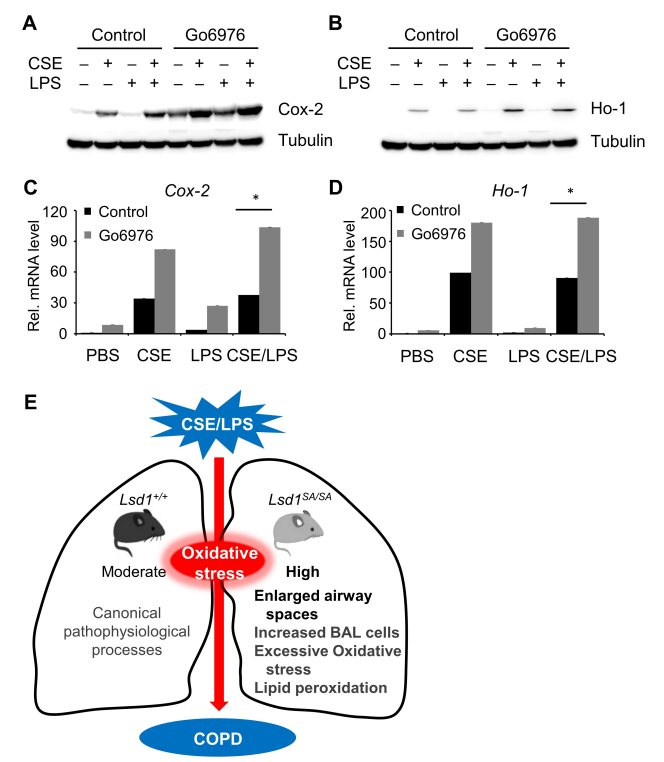

Fig. 4. Effect of Go6976 on CSE/LPS-induced Cox-2 and Ho-1 expressions in $L s d 1^{S A / S A}$ MEFs. (A, B) Immunoblot analysis using the indicated antibodies in $W T$ and $L s d 1^{\text {SA/SA }}$ MEFs exposed to CSE and LPS for $24 \mathrm{~h}$ following $10 \mu \mathrm{M}$ Go6976 pre-treatment for 1 h. (C, D) Quantitative RT-PCR analysis of Cox-2 and Ho-1 mRNAs in $W T$ and $L s d 1^{S A / S A}$ MEFs treated with $10 \mu \mathrm{M}$ Go6976 for $1 \mathrm{~h}$ prior to incubation with CSE and LPS for $4 \mathrm{~h}$. (E) The illustration summarizing the conclusions of this study. Lsd1 ${ }^{\text {SA/SA }}$ mice show excessive inflammation, oxidative stress, and lung damage following CSE/LPS exposure, resulting in exacerbation of COPD. All results represent three independent experiments. Data are presented as mean $\pm \mathrm{SEM} ; * \mathrm{P}<0.05$.
CSE, LPS, or CSE/LPS for $4 \mathrm{~h}$ to evaluate the Cox-2 and Ho-1 mRNA levels. Pre-treatment with Go6976 markedly increased Cox-2 and Ho-1 mRNA expression (Fig. 4C, D). These data indicate that inhibition of Lsd1-S112 phosphorylation up-regulated CSE-induced Cox-2 and Ho-1 transcription. Our data suggest that the Lsd1-S112A mutation and Go6976 treatment indeed strengthen CSE and LPS-induced Cox-2 and Ho-1 protein expression. Taken together, our findings reveal that the phosphorylation defect on Lsd1-S112 enhances inflammatory and oxidative stress responses and exacerbates CSE/LPS-induced COPD (Fig. 4E).

\section{DISCUSSION}

LSD1 is an epigenetic regulator that controls lysine methylation status of histones and non-histone proteins, and its biological functions are regulated by post-translational modifications including phosphorylation $(12,15,17,18)$. Emerging evidence indicates that phosphorylation of LSD1 at serine 112 (equivalent to serine 111 on the human protein) plays a crucial role in the regulation of inflammatory responses in sepsis and IBD models $(15,19)$. To the best of our knowledge, this is the first study to demonstrate the effect of Lsd1-S112A on the murine CSE/LPS-induced COPD model.

Chronic inflammation and oxidative stress are important features of the pathogenesis of COPD. CS, a potent inducer of neutrophilic inflammation, alters the inflammatory responses by modulating the production of cytokines and chemokines with consecutive recruitment of macrophages and neutrophils, and thus, causing further damage to the lung tissue (25). CS is a major exogenous source of oxidative stress in the lung. Oxidative stress is implicated in airway inflammation and is one of the major prognostic factors in the pathogenesis of COPD. High levels of ROS are generated by neutrophils following CS exposure and contribute to oxidative stress (21). In the current study, we showed that intratracheal instillation of CSE combined with LPS led to increased immune cell influx and more severe parenchymal destruction in $L s d 1^{\text {SA/SA }}$ mice compared with that in WT mice. $L s d 1^{S A / S A}$ mice exhibited higher expression of both Cox-2 and Ho-1 proteins upon CSE/LPS exposure which contributed to airway remodeling and inflammation. Furthermore, several oxidative stress products, such as 4-HNE and Ho-1, were also increased in the Lsd1 ${ }^{\text {SAISA }}$ MEFs after CSE/LPS treatment. Lung tissues from patients with COPD show increased lipid peroxidation, as measured by 4-HNE levels (26). Lipid peroxidation products, such as Ox-PLs and 4-HNE are known to mediate cytotoxicity by disrupting membrane integrity and activating cell death pathways including apoptosis and ferroptosis (27). Our results indicate that $L s d 1-S 112 A$ mice are more susceptible to inflammation and oxidative stress in response to CSE and LPS, compared with WT mice. This suggests that phosphorylation defect of LSD1 on S112 is involved in exacerbating lung damage. Further investigations are required to understand the 
underlying mechanism how LSD1 phosphorylation contributes to the restriction of oxidative stress in CSE-induced response. A recent study revealed that the transcriptional activity of LSD1 is modulated by chromatin remodeling, and depends on steady ROS levels and redox signaling (28). It is also reported that the changes in the redox environment increases LSD1 activity in DNA repair after oxidative damage (29). We speculate that phosphorylation-defective Lsd1-S112A is involved in excessive oxidative stress, leading to lung damage due to endogenous ROS.

Taken together, our findings reveal that the phosphorylation defect on Lsd1-S112 is critical for inflammation and oxidative stress upon CSE/LPS exposure and plays a key role in the exacerbation of COPD. This study provides new insights into the correlation between LSD1 phosphorylation and oxidative stress in exacerbating COPD.

\section{MATERIALS AND METHODS}

\section{Animals}

Generation of $L s d 1^{S A / S A}$ mice of the C57BL/6] strain have been described previously (12). WT and $L s d 1^{S A / S A}$ female mice aged 8-10 weeks were used in this study. All the animal experiments were conducted strictly in accordance with the protocols approved by the Institutional Animal Care and Use Committee (IACUC) of Sookmyung Women's University (SMWU-IACUC1711-029).

\section{Preparation of CSE}

We used THIS cigarettes (KT\&G, Korea), and each cigarette contained $1.1 \mathrm{mg}$ nicotine, $15 \mathrm{mg}$ tar, and $15 \mathrm{mg}$ carbon monoxide. Briefly, the smoke from five cigarettes was bubbled slowly through $100 \mathrm{ml}$ phosphate-buffered saline using a vacuum pump, which was considered as a $100 \%$ CSE solution. The soluble CSE was then sterilized and stored at $-80^{\circ} \mathrm{C}$.

\section{CSE and LPS-induced COPD murine model}

Mice were treated with CSE, LPS or CSE/LPS solutions for establishing the COPD murine model (Fig. 1). Briefly, the solutions were administered by intratracheal injection 4 times at 7-day intervals. The mice were divided into four groups as follows; control group (100 $\mu$ PBS), CSE group (100 $\mu \mathrm{l} \mathrm{CSE),}$ LPS group (10 $\mu \mathrm{g}$ LPS in $100 \mu \mathrm{l} \mathrm{PBS})$ and CSE/LPS group (10 $\mu \mathrm{g}$ LPS in $100 \mu \mathrm{l} \mathrm{CSE}$ ). The mice were euthanized at day 7 or $24 \mathrm{~h}$ after the last dose.

\section{Analysis of bronchoalveolar lavage fluid (BALF)}

BALF was obtained through the tracheal cannula by washing the airway lumen with $1 \mathrm{ml}$ of sterile saline. Total cell counts in the BALF were determined using a hemocytometer after lysis of red blood cells with a red blood cell lysis buffer (Sigma, USA). Differential cell counts in the BALF were examined by Cytospin preparation followed by staining with the Diff-Quik reagent (Sysmex International Reagents, Kobe, Japan).

\section{Histological analysis}

The left lung tissue of each mouse was fixed with an intratracheal injection of $4 \%$ paraformaldehyde and immersed in the same fixative to preserve pulmonary architecture. The fixed lungs were then embedded in paraffin using standard procedures. Lung sections $(4 \mu \mathrm{m})$ were stained with H\&E for histological examinations. MLI and inflammation score were determined blindly by two independent observers (30).

\section{Cell culture and treatments}

MEFs were cultured in DMEM (Gibco) supplemented with $10 \%$ fetal bovine serum (Gibco), 1\% Penicillin/streptomycin (Gibco). All cells tested negative for the presence of mycoplasma before experiments. The cells were treated with $10 \%$ CSE and $1 \mu \mathrm{g} / \mathrm{ml}$ LPS for the indicated time periods.

\section{Immunoblotting}

Lung tissue and cells were lysed in RIPA lysis buffer supplemented with protease and phosphatase inhibitors (GenDEPOT). Total protein was quantified using the Bradford assay (Bio-Rad) according to the manufacturer's instructions and subjected to SDS-PAGE. Primary antibodies used were anti-HO-1 (sc-136960), anti-COX-2 (CST, \#12282), anti-Tubulin (Sigma, T6074), anti-GAPDH (sc-25778), and anti-4HNE (Abcam, ab46545). Specific bands were quantified by densitometry using NIH ImageJ.

\section{Real-time RT-PCR}

Total RNA was isolated using the TRIzol reagent (ThermoFisher Scientific) according to the manufacturer's protocol. The complementary DNA (CDNA) was synthesized from $1 \mu \mathrm{g}$ of total RNA using the cDNA synthesis kit (Thermo-Fisher Scientific). Quantitative PCR was performed using SYBR Green (Applied Biosystems) and detected on the StepOnePlus ${ }^{\mathrm{TM}}$ Real-Time PCR system (Applied Biosystems). Primers used for the mouse genes are as follows. Ho-1: 5'-GGTGATGGCTTCCTTGTACC-3' (FW), 5'-AGTGAGGCCCATACCAGA AG-3' (RV), Cox2: 5'-AACCGCATTGCCTCTGAAT-3' (FW), 5'-CATGTTCCAGGAGGAT GGAG-3' (RV), Gapdh: 5'-TCCCACTCTTCCACCTTCGA-3' (FW), 5'-AGTTGGGATAGG GCCTCT CTTG-3' (RV).

\section{Statistical analysis}

All values are shown as mean \pm standard error of mean (SEM). Differences between groups were examined for statistical significance using one-way analysis of variance (ANOVA) and then determined with the least significant difference test. The criterion for significance was $\mathrm{P}<0.05$.

\section{ACKNOWLEDGEMENTS}

This work was supported by Basic Science Research Program (NRF-2018R1A2B6004112) to K.I.K. from the National Research Foundation of Korea (NRF), and by the Center for Women In 
Science, Engineering and Technology (WISET) Grant to J.J. from the Program for Returners into R\&D, funded by the Ministry of Science and ICT (MSIT).

\section{CONFLICTS OF INTEREST}

The authors have no conflicting interests.

\section{REFERENCES}

1. Corlateanu A, Mendez Y, Wang Y et al (2020) Chronic obstructive pulmonary disease and phenotypes: a state-ofthe-art. Pulmonology 26, 95-100

2. Sundar IK and Rahman I (2016) Gene expression profiling of epigenetic chromatin modification enzymes and histone marks by cigarette smoke: implications for COPD and lung cancer. Am J Physiol Lung Cell Mol Physiol 311, L1245-L1258

3. Ito K, Ito M, Elliott WM et al (2005) Decreased histone deacetylase activity in chronic obstructive pulmonary disease. N Engl J Med 352, 1967-1976

4. Nakamaru Y, Vuppusetty C, Wada H et al (2009) A protein deacetylase SIRT1 is a negative regulator of metalloproteinase-9. FASEB J 23, 2810-2819

5. Sundar IK, Nevid MZ, Friedman AE et al (2014) Cigarette smoke induces distinct histone modifications in lung cells: Implications for the pathogenesis of COPD and lung cancer. J Proteome Res 13, 982-996

6. Stepaniants S, Wang I, Boie Y et al (2014) Genes related to emphysema are enriched for ubiquitination pathways. BMC Pulm Med 14, 187

7. Liu W, Zhang Q, Fang Y et al (2018) The deubiquitinase USP38 affects cellular functions through interacting with LSD1. Biol Res 51, 53

8. Forneris F, Binda C, Vanoni MA et al (2005) Histone demethylation catalysed by LSD1 is a flavin-dependent oxidative process. FEBS Lett 579, 2203-2207

9. Shi Y, Lan F, Matson C et al (2004) Histone demethylation mediated by the nuclear amine oxidase homolog LSD1. Cell 119, 941-953

10. Maiques-Diaz A and Somervaille TC (2016) LSD1: biologic roles and therapeutic targeting. Epigenomics 8, 1103

11. Amente S, Lania L and Majello B (2013) The histone LSD1 demethylase in stemness and cancer transcription programs. Biochim Biophys Acta 1829, 981-986

12. Nam HJ, Boo K, Kim D et al (2014) Phosphorylation of LSD1 by PKC $\alpha$ is crucial for circadian rhythmicity and phase resetting. Mol Cell 53, 791-805

13. Peng B, Shi R, Jiang W et al (2017) Phosphorylation of LSD 1 by PLK 1 promotes its chromatin release during mitosis. Cell Biosci 7, 15

14. Peng B, Wang J, Hu Y et al (2015) Modulation of LSD1 phosphorylation by CK2/WIP1 regulates RNF168-dependent 53BP1 recruitment in response to DNA damage. Nucleic Acids Res 43, 5936-5947

15. Kim D, Nam HI, Lee W et al (2018) PKC $\alpha-L S D 1-N F-\kappa B-$ signaling cascade is crucial for epigenetic control of the inflammatory response. Mol Cell 69, 398-411 e396

16. Tsai CL, Chao AS, Jung SM, et al (2018) Stress-induced phosphoprotein 1 acts as a scaffold protein for glycogen synthase kinase-3ß-mediated phosphorylation of lysinespecific demethylase 1 . Oncogenesis 7,31

17. Lim CS, Nam HI, Lee J et al (2017) PKC $\alpha$-mediated phosphorylation of LSD1 is required for presynaptic plasticity and hippocampal learning and memory. Sci Rep 7, 4912

18. Feng J, Xu G, Liu J et al (2016) Phosphorylation of LSD1 at Ser112 is crucial for its function in induction of EMT and metastasis in breast cancer. Breast Cancer Res Treat 159, 443-456

19. Oh C, Jeong J, Oh SK et al (2020) Inhibition of LSD1 phosphorylation alleviates colitis symptoms induced by dextran sulfate sodiumlnhibition of LSD1 phosphorylation alleviates colitis symptoms induced by dextran sulfate sodium. BMB Rep 53, 385-390

20. Naito M, Taguchi O, Kobayashi T et al (2013) Thrombinactivatable fibrinolysis inhibitor protects against acute lung injury by inhibiting the complement system. Am J Respir Cell Mol Biol 49, 646-653

21. Strzelak A, Ratajczak A, Adamiec A et al (2018) Tobacco smoke induces and alters immune responses in the lung triggering inflammation, allergy, asthma and other lung diseases: a mechanistic review. Int J Environ Res Public Health 15, 1033

22. Yu D, Liu X, Zhang G et al (2018) Isoliquiritigenin inhibits cigarette smoke-induced COPD by attenuating inflammation and oxidative stress via the regulation of the Nrf2 and NF- $\mathrm{kB}$ signaling pathways. Front Pharmacol 9, 1001

23. Zarkovic N (2003) 4-Hydroxynonenal as a bioactive marker of pathophysiological processes. Mol Aspects Med 24, 281-291

24. Ayala A, Muñoz MF and Argüelles S (2014) Lipid peroxidation: production, metabolism, and signaling mechanisms of malondialdehyde and 4-hydroxy-2-nonenal. Oxid Med Cell Longev 2014, 360438

25. Churg A, Zay K, Shay S et al (2002) Acute cigarette smoke-induced connective tissue breakdown requires both neutrophils and macrophage metalloelastase in mice. Am J Respir Cell Mol Biol 27, 368-374

26. Rahman I, van Schadewijk AA, Crowther AJ et al (2002) 4-Hydroxy-2-nonenal, a specific lipid peroxidation product, is elevated in lungs of patients with chronic obstructive pulmonary disease. Am J Respir Crit Care Med 166, 490-495

27. Gaschler MM and Stockwell BR (2017) Lipid peroxidation in cell death. Biochem Biophys Res Commun 482, 419-425

28. Pezone A, Taddei ML, Tramontano A et al (2020) Targeted DNA oxidation by LSD1-SMAD2/3 primes TGF- $31 /$ EMT genes for activation or repression. Nucleic Acids Res 48, 8943-8958

29. Duquette ML, Kim J, Shi LZ et al (2018) LSD1 mediated changes in the local redox environment during the DNA damage response. PLoS One 13, e0201907

30. Braber S, Henricks PA, Nijkamp FP et al (2010) Inflammatory changes in the airways of mice caused by cigarette smoke exposure are only partially reversed after smoking cessation. Respir Res 11, 99 